\title{
Acumulación de cobre en una comunidad vegetal afectada por contaminación minera en el valle de Puchuncaví, Chile central
}

\author{
Copper accumulation in a plant community affected by mining contamination in \\ Puchuncaví valley, central Chile
}

ISABEL GONZÁLEZ ${ }^{1}$, VICTORIA MUENA ${ }^{1}$, MAURICIO CISTERNAS $^{2} \&$ ALEXANDER NEAMAN $^{3,4}$, *

\author{
${ }^{1}$ Programa de Magíster en Producción Agroambiental, Facultad de Agronomía, \\ Pontificia Universidad Católica de Valparaíso, Chile \\ 2 Área de Medio Ambiente, Laboratorio de Fitogenética, Facultad de Agronomía, Pontificia Universidad Católica \\ de Valparaíso, Chile \\ ${ }^{3}$ Facultad de Agronomía, Pontificia Universidad Católica de Valparaíso, Chile \\ ${ }^{4}$ Centro Regional de Estudios en Alimentos Saludables (CREAS), Región de Valparaíso, Chile \\ *e-mail para correspondencia: alexander.neaman@ucv.cl
}

\begin{abstract}
RESUMEN
Las especies hiperacumuladoras son capaces de acumular más de $1.000 \mathrm{mg} \mathrm{kg}^{-1}$ de metal en su biomasa aérea y son útiles en procesos de fitoextracción de metales en suelos contaminados por actividades mineras. Con el fin de identificar especies hiperacumuladoras representativas de las condiciones chilenas, se realizó una prospección dentro de la diversidad vegetal en el área afectada por las emisiones de la Fundición Ventanas (90-900 $\mathrm{mg} \mathrm{kg}^{-1}$ de $\mathrm{Cu}$ total en suelos), así como en un área cercana a una pila de escorias de fundición (500-3.000 mg kg-1 de Cu total en suelos). Se determinaron las concentraciones de $\mathrm{Cu}$ en la biomasa aérea de las plantas. Los resultados indican que dentro de la diversidad del sitio existen al menos veintidós especies pseudometalofitas, es decir, ecotipos de especies comunes que son capaces de tolerar concentraciones de cobre en el suelo que para una planta normal serían tóxicas. Las especies fueron clasificadas según su concentración de cobre y mostraron en su mayoría media (200-600 mg kg-1) o baja (< $200 \mathrm{mg} \mathrm{kg}^{-1}$ ) acumulación de cobre. La especie con la concentración más alta de cobre fue Oenothera affinis $\left(614 \mathrm{mg} \mathrm{kg}^{-1}\right)$. Sin embargo, no se identificaron hiperacumuladoras. Oenothera affinis podría considerarse como una buena candidata para iniciativas de remediación en Chile, ya que es una hierba nativa perenne, resistente a la sequía, fácil de propagar y que produce una gran biomasa. Sin embargo, es necesario comprobar que no hubo alguna sobreestimación de las concentraciones de $\mathrm{Cu}$ en las plantas, debido a la adherencia de partículas contaminantes en los tricomas. Además, se realizaron dos nuevos muestreos más tarde en la temporada, para Argemone subfusiformis y Oenothera affinis. Existe una disminución en la concentración de $\mathrm{Cu}$ en la biomasa aérea al avanzar la temporada causada por el desarrollo de estructuras de baja acumulación de $\mathrm{Cu}$ (flores, frutos y hojas de verano) en el estado reproductivo y a la pérdida de la biomasa de alta concentración de $\mathrm{Cu}$ al llegar a la senescencia. Además, este último muestreo sugiere que pudo existir un grado de retranslocación de cobre desde la biomasa aérea hacia las estructuras subterráneas durante la senescencia, en $O$. affinis.
\end{abstract}

Palabras clave: Oenothera affinis, metalofitas, cobre, fitoextracción.

\begin{abstract}
Hyperaccumulator plants species are capable of accumulating more than $1,000 \mathrm{mg} \mathrm{Cu} \mathrm{kg}^{-1}$ in their shoots and are useful for metal phytoextraction in soils contaminated by mining activities. To identify the hyperaccumulator plants representative of the Chilean conditions, we carried out a survey of plant diversity in the area affected by the emissions of the Ventanas smelter (90-900 $\mathrm{mg} \mathrm{kg}^{-1}$ of total $\mathrm{Cu}$ in soils) and in a nearby area close to a smelter slug pile $\left(500-3,000 \mathrm{mg} \mathrm{kg}^{-1}\right.$ of total $\mathrm{Cu}$ in soils). Copper concentrations in the shoots of the studied plants were determined. Results indicate that there were at least twenty-two pseudometallophyte species, i.e., ecotypes of common species capable to tolerate concentrations of $\mathrm{Cu}$ in the soil that would be toxic for a normal plant. The species were classified by their copper accumulation and nearly all exhibited medium $\left(200-600 \mathrm{mg} \mathrm{kg}^{-1}\right)$ or low $\left(<200 \mathrm{mg} \mathrm{kg}^{-1}\right)$ accumulation of copper. The species with the highest accumulation of copper was Oenothera affinis $\left(614 \mathrm{mg} \mathrm{kg}^{-1}\right)$. However, no hyperaccumulator species were found. Oenothera affinis could be considered as a good candidate for
\end{abstract}


remediation initiatives in Chile, because it is a native perennial herb, it is drought resistant, it is easy to propagate, and that it produces a large biomass. However, it is still required to verify that there was no overestimation of $\mathrm{Cu}$ concentrations in the plants due to attachment of particles onto the trichomes. In addition, two new samplings were carried out later in the growing season, for Argemone subfusiformis and Oenothera affinis. There was a decrease in the concentration of $\mathrm{Cu}$ in the shoots with the advance of the season caused by the development of structures with low $\mathrm{Cu}$ accumulation (flowers, fruits, and summer leaves) during the reproductive stage and the loss of $\mathrm{Cu}$-rich biomass during the senescence. Moreover, this last sampling suggests that there was possibly a certain re-translocation of $\mathrm{Cu}$ into the subterranean structures during the senescence for $O$. affinis.

Key words: Oenothera affinis, metallophytes, copper, phytoextraction.

\section{INTRODUCCIÓN}

En sitios cercanos a minas y fundiciones de metales es común encontrar extensas áreas contaminadas con diversos elementos ecotóxicos, tales como: cobre, cadmio, plomo, arsénico y otros. La mayoría de las plantas no crecen en estos sitios, ya que las altas concentraciones de estos elementos son tóxicas para sus organismos. Sin embargo, existe un grupo de plantas denominadas metalofitas, que son capaces de desarrollarse en estas condiciones. Este grupo de plantas incluye a las metalofitas estrictas, es decir, aquellas que solo crecen en sitios contaminados (endémicas) y a las pseudometalofitas, es decir, poblaciones tolerantes de especies comunes (Pollard et al. 2002). Dentro del grupo de las metalofitas, existe un grupo aún más reducido denominado hiperacumuladoras, definidas como especies metalofitas capaces de concentrar metales. En el caso de $\mathrm{Cu}$, estas plantas pueden acumular más de $1.000 \mathrm{mg} \mathrm{kg}^{-1}$ del contaminante en su biomasa aérea en base a su materia seca (Baker \& Brooks 1989, McGrath et al. 1993).

Las especies hiperacumuladoras han adquirido relevancia en las últimas décadas, debido a su potencial uso en técnicas de fitoextracción de metales en suelos contaminados. Esta técnica consiste en cultivar especies que acumulan un determinado contaminante por una o varias temporadas. Posteriormente, la biomasa aérea es cosechada e incinerada y las cenizas restantes son tratadas como desechos peligrosos (Ginocchio 2004).

La eficiencia de un proceso de fitoextracción depende principalmente de la selección de una especie adecuada. Con este fin, se debe considerar que la capacidad de fitoextracción de una planta depende tanto de su conformación genética como de las condiciones ambientales a las que está sometida (Pollard et al. 2002). La especie ideal debería estar adaptada a las condiciones edafoclimáticas del sitio a remediar, ya que esto le permite un correcto desarrollo vegetativo que facilita los procesos de remoción y translocación de los contaminantes (Vara \& Oliveira 2003, Ginocchio 2004).

La realización de una prospección de especies acumuladoras, dentro de la diversidad vegetal existente en los mismos sitios que se desea remediar, aseguraría trabajar con especies totalmente adaptadas a las condiciones climáticas y edáficas. Dado que en estas áreas solamente se desarrollan especies metalofitas, es posible que existan algunas especies que además de resistentes sean acumuladoras. Investigaciones de este tipo han sido reportadas por ejemplo en suelos serpentinos en Turquía donde se encontraron, dentro de 45 especies, 13 hiperacumuladoras de Ni del género Centaurea (Reeves \& Adigüzel 2004). En Katanga (Congo), en una afloración de cobre/cobalto, se identificó de entre 76 especies metalofitas, a Aeollanthus subacaulis var. ericoides, hiperacumuladora de cobalto (Malaisse et al. 1999). También, en Nueva Caledonia, entre 32 especies que crecían en una afloración de níquel, se identificaron 12 especies hiperacumuladoras de este metal de la familia Flacourticeae (Jaffré et al. 1979).

En Chile, a pesar de la alta diversidad de especies y la gran cantidad de explotaciones mineras existentes, se han identificado solamente seis especies metalofitas para $\mathrm{Cu}$ (Mimulus luteus var. variegatus, Cenchrus echinatus, Erygeron berterianum, Mullinum spinosum, Nolana divaricata, y Dactylium sp.), pero aún no se han reconocido especies hiperacumuladoras (Ginocchio \& Baker 2004).

La localidad de Los Maitenes en el valle de Puchuncaví (Chile central) es un área altamente contaminada por $\mathrm{Cu}$, debido a que recibió por 
más de 35 años las descargas de emisiones gaseosas emitidas por la Fundición Ventanas (Bruno 1992). Actualmente, en Chile no existe una legislación sobre las concentraciones máximas permitidas de elementos tóxicos en los suelos. Por lo tanto, se deben usar legislaciones internacionales como referencia. Por ejemplo, la Swedish Environmental Protection Agency (disponible en www.internat.naturvardsverket.se) postula que los suelos con una concentración total de cobre superior a $100 \mathrm{mg} \mathrm{kg}^{-1}$ deben ser considerados como contaminados. Según los datos obtenidos por De Gregori et al. (2003), las concentraciones de $\mathrm{Cu}$ total en Los Maitenes (104 a $530 \mathrm{mg} \mathrm{kg}^{-1}$ ) sobrepasan hasta cinco veces estos estándares. Sin embargo, se debe tener en cuenta que dichos estándares no consideran las altas concentraciones de fondo que existen en Chile para $\mathrm{Cu}$ (R. Aguilar, resultados no publicados).

Ginocchio (2000) reportó efectos negativos asociados a las emisiones de material particulado y $\mathrm{SO}_{2}$ de la Fundición Ventanas, en las comunidades de matorrales silvestres en el valle de Puchuncaví. Actualmente, la vegetación del valle se compone de algunas poblaciones de las especies que existían originalmente, las cuales han desarrollado mecanismos de tolerancia a las actuales condiciones edáficas (Ginocchio 1997).

En base a los antecedentes, se postula que es posible encontrar especies metalofitas dentro de la diversidad vegetal de la localidad de Los Maitenes y, entre ellas, especies hiperacumuladoras de $\mathrm{Cu}$. El objetivo de esta investigación fue identificar especies vegetales capaces de acumular cobre en cantidades sobre lo normal. Para este propósito, se escogieron dos sitios en las cercanías de la Fundición Ventanas, donde se realizó una prospección de plantas en su hábitat natural.

\section{MATERIALES Y MÉTODOS}

\section{Sitios de muestreo}

La prospección de especies se llevó a cabo en un área ubicada aproximadamente a $3 \mathrm{~km}$ al sur-este de la Fundición Ventanas (UTM 6372975 N, 267350 E), en la localidad de Los
Maitenes, comuna de Puchuncaví, Región de Valparaíso, Chile (Fig. 1). Se seleccionaron dos áreas representativas, ambas con concentraciones de cobre sobre lo normal según la Swedish EPA (disponible en www.internat.naturvardsverket.se). La primera corresponde a un área de matorral típico de la zona (UTM 6371295 N, 268650 E, Fig. 1) ubicada al norte y al sur del camino a Los Maitenes, con predominancia de una cobertura herbácea y matorrales (Ginocchio et al. 2004). Esta área fue considerada como representativa de la zona que ha recibido la contaminación atmosférica proveniente de la fundición. La segunda área con concentraciones mayores de cobre que el área anterior, se encuentra en las cercanías de una pila de escorias de fundición (propiedad de la minera Tocopilla, UTM 6370072 N, 269355 E, Fig. 1). Esta área es considerada como representativa de los suelos contaminados por depósitos mineros. En esta área la cobertura vegetal disminuye gradualmente hasta desaparecer totalmente al pie de la pila de escorias de fundición.

\section{Prospección de especies}

La prospección e identificación de especies fue realizada en el mes de agosto de 2005. En cada una de las áreas de muestreo, se recolectó biomasa aérea de las especies herbáceas y arbustivas. Para la identificación de las especies vegetales, se utilizaron los trabajos de Johnston (1947), Navas (1979), Marticorena \& Quezada (1985), Matthei (1995), y Leuenberger \& Eggli (2002).

Para cada especie identificada, se muestrearon dos individuos, ubicados a no más de $3 \mathrm{~m}$ de distancia entre ellos. Ambos individuos fueron considerados como una misma muestra y representativos de un mismo suelo. En cada sitio se tomaron con barreno cinco muestras al azar de $1-1,5 \mathrm{~kg}$ de suelo, desde la superficie hasta unos $20 \mathrm{~cm}$ de profundidad. Las muestras fueron trasladadas al laboratorio en cartuchos de papel.

Muestreo para el análisis de la variación temporal de cobre en algunas especies

Para estudiar la variación estacional de la concentración de $\mathrm{Cu}$ en la biomasa aérea de las especies, se consideró el muestreo de agosto 
(especies en estado vegetativo) y se realizó un nuevo muestreo en diciembre de 2005 (especies en estado reproductivo) para las especies Chenopodium ambrosioides, Sphaeralcea velutina, Verbascum virgatum, Oenothera affinis y Argemone subfusiformis. Cada especie se muestreó en el mismo sector que en agosto. En un tercer muestreo en marzo del 2006 (especies en senescencia), se muestreó solamente Oenothera affinis.

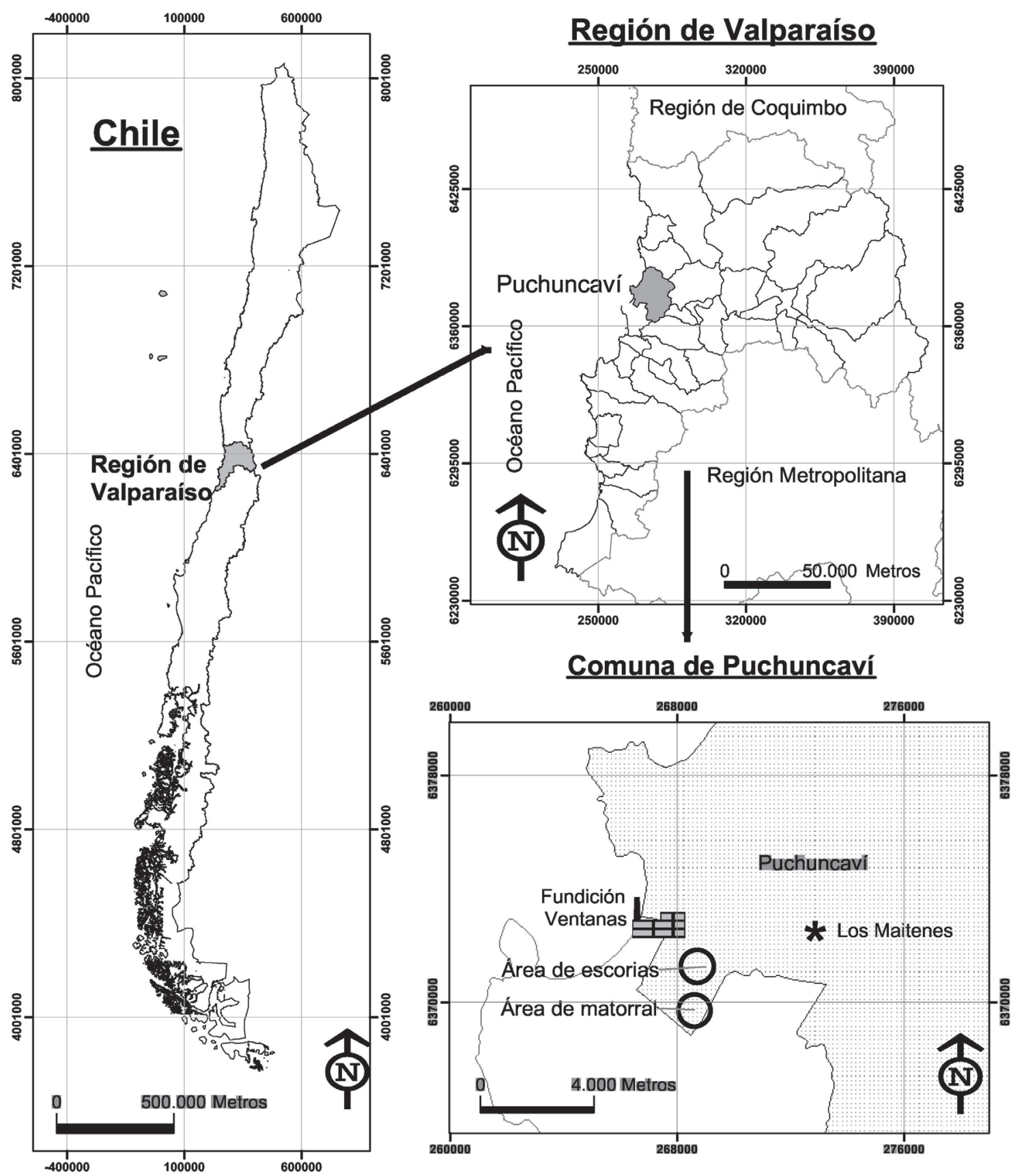

Fig.1: Ubicación geográfica del área de estudio.

Geographical location of the study area. 


\section{Análisis de suelos}

Las concentraciones de $\mathrm{Cu}$ total en los suelos fueron determinadas mediante espectrometría de absorción atómica, luego de la digestión ácida de las muestras usando una mezcla de ácidos fluorhídrico y perclórico (Maxwell 1968). Se analizaron independientemente las cinco muestras representativas de cada sitio de muestreo, considerándose dos repeticiones para cada una de ellas. Para el aseguramiento y control de calidad de los resultados, se analizaron dos muestras de referencia: GBW 07312 (sedimento de río) y IAEA-SL-1 (sedimento lacustre) del International Atomic Energy Agency. Los valores de referencia y los obtenidos se presentan en la Tabla 1.

Según Kabata-Pendias (2004), la extracciones con sales neutras en concentraciones relativamente bajas son consideradas como las mejores para simular las concentraciones fitodisponibles de elementos traza en el suelo. En esta investigación, se utilizó $\mathrm{KNO}_{3} 0,1 \mathrm{M}$ como extractante (razón suelo: solución de 1: 2,5). En adelante, esta fracción extraída se denominará como "soluble". La concentración de $\mathrm{Cu}$ soluble se determinó mediante espectroscopia de absorción atómica. El pH se determinó en la misma solución de $\mathrm{KNO}_{3} 0,1 \mathrm{M}$.

\section{Análisis de cobre en las plantas}

Las plantas muestreadas en agosto fueron lavadas dos veces con agua potable para remover partículas de suelo y posteriormente dos veces con agua destilada (Tang et al. 1999, Poschenrieder et al. 2001). El material vegetal se secó por $48 \mathrm{~h}$ en una estufa a $60{ }^{\circ} \mathrm{C}$, triturándose luego en molino de acero inoxidable. Para evitar la contaminación cruzada de las muestras, el molino se limpió con aire comprimido entre una muestra y otra.

Para realizar los análisis de cada muestra compuesta (de dos individuos de la misma especie), se obtuvieron tres submuestras (repeticiones). El material vegetal triturado fue digerido en matraces Erlenmayer con una mezcla de ácidos nítrico y perclórico concentrados, siguiendo el método de Soon (1998). Posteriormente, las concentraciones de cobre en las soluciones fueron determinadas por espectroscopia de absorción atómica.

Para comprobar la exactitud y precisión del método, se llevaron a cabo análisis de dos muestras de referencia: 1570a (espinaca) y 1573a (tomate) del National Institute of Standards and Technology. Los valores obtenidos y los de referencia se presentan en la Tabla 1.

Posteriormente, las especies se clasificaron según la concentración de $\mathrm{Cu}$ en sus biomasas aéreas, en rangos sugeridos por los autores: baja acumulación $\left(<200 \mathrm{mg} \mathrm{kg}^{-1}\right)$, media acumulación (200-600 $\mathrm{mg} \mathrm{kg}^{-1}$ ) y alta acumulación (601-1,000 mg kg-1). Se consideró que una planta con una concentración mayor a $1.000 \mathrm{mg} \mathrm{kg}^{-1}$ es hiperacumuladora (Baker \& Brooks 1989). Además, concentraciones de $\mathrm{Cu}$ menores o iguales a $20 \mathrm{mg} \mathrm{kg}^{-1}$ se consideraron como normales en la biomasa aérea de una planta (Adriano 1986).

Para las plantas muestreadas en diciembre y marzo (segundo y tercer muestreo), la biomasa aérea fue separada en hojas de otoño (en la parte baja del tallo), hojas de verano (en la parte alta del tallo), tallo, flores y frutos, siguiéndose el mismo procedimiento anterior para el análisis de $\mathrm{Cu}$.

\section{TABLA 1}

Resultados del análisis de muestras de referencia para $\mathrm{Cu}$. Se presenta el valor promedio $(\mathrm{n}=5)$, la desviación estándar y el porcentaje de diferencia entre el valor obtenido versus el certificado

Results of the analysis of $\mathrm{Cu}$ in the reference materials. Average $(\mathrm{n}=5)$, standard deviation, and the percentage of difference between the determined value versus the reference value are shown

\begin{tabular}{lcccc}
\hline & $\begin{array}{c}\text { Espinaca 1570a } \\
\left(\mathrm{mg} \mathrm{kg}^{-1}\right)\end{array}$ & $\begin{array}{c}\text { Tomate 1573a } \\
\left(\mathrm{mg} \mathrm{kg}^{-1}\right)\end{array}$ & $\begin{array}{c}\text { Sedimento de río } \\
\text { GBW 07312 }\left(\mathrm{mg} \mathrm{kg}^{-1}\right)\end{array}$ & $\begin{array}{c}\text { Sedimento lacustre } \\
\text { IAEA-SL-1 }\left(\mathrm{mg} \mathrm{kg}^{-1}\right)\end{array}$ \\
\hline Valor certificado & $12,2 \pm 0,60$ & $4,70 \pm 0,14$ & $1.230 \pm 12$ & $30,0 \pm 5,0$ \\
Valor obtenido & $11,6 \pm 0,41$ & $4,58 \pm 0,12$ & $1.250 \pm 14$ & $28,8 \pm 0,7$ \\
Diferencia, \% & 4,8 & 2,7 & 1,6 & 4,7 \\
\hline
\end{tabular}




\section{Análisis estadístico de los datos}

La relación entre el $\mathrm{Cu}$ total y soluble y el $\mathrm{pH}$ del suelo fue analizada por regresión múltiple $(\mathrm{P}<0,05)$. Se utilizó el Software MINITAB 13.1 para Windows.

\section{RESULTADOS Y DISCUSIÓN}

\section{Caracterización de los suelos}

Se encontraron fuertes variaciones espaciales en las concentraciones de cobre en los suelos estudiados (Tabla 2), al igual que lo reportado anteriormente por Ginocchio et al. (2004). Los promedios para $\mathrm{Cu}$ total y soluble para los suelos del área de escorias de fundición son mayores que para los suelos del área de matorral, dado que la pila de escoria se compone de desechos del proceso productivo, los que hacen que su composición sea alta en $\mathrm{Cu}$ y otros elementos. Los suelos en ambos sitios presentaron valores de pH en el rango de 3,9-5,9.

Los resultados de la regresión simple entre cobre total y soluble indican una relación significativa $(\mathrm{P}<0,05 ; \mathrm{n}=13)$, pero el modelo solo satisface un $51 \%$ de la varianza. Al agregar la variable $\mathrm{pH}$ al modelo, y luego de aplicar transformación logarítmica a los datos, la regresión arrojó una correlación significativa entre las tres variables $(\mathrm{P}<0,001)$ y el modelo es capaz de satisfacer el $84 \%$ de la varianza: Log $\mathrm{Cu}_{\text {soluble }}=1,63+0,969 \log \mathrm{Cu}_{\text {total }}-0,704 \mathrm{pH}$.

\section{Especies identificadas}

Se identificaron en total 22 especies (Tabla 3 ). Sin embargo, estas especies no representan la diversidad vegetal existente en el sector, ya que solamente se muestrearon especies con biomasa adecuada para un proceso de fitoextracción, excluyéndose así las especies herbáceas de menor tamaño (por ejemplo, gramíneas).

Siete de las especies muestreadas fueron reconocidas exclusivamente en el área de las escorias y seis exclusivamente en el área de matorral. Nueve especies se muestrearon en ambas áreas. La familia Asteraceae fue la más abundante con seis especies. La mayor parte de las especies corresponden a hierbas anuales (terófitas, ocho especies), bianuales (terófitas, dos especies) o perennes (criptófitas o hemicriptófitas, siete especies), el resto corresponden a arbustos (nanofanerófitas, cinco especies). En cuanto al origen de las especies, nueve especies son introducidas y once son nativas, entre estas últimas Sphaeralcea velutina, Atriplex repanda y Astragalus amatus son consideradas endémicas (Tabla 3).

En relación al estado fenológico, la mayor parte de las especies muestreadas en agosto se encontraban en estado de desarrollo vegetativo, el resto presentaban estructuras reproductivas, en su mayoría únicamente flores (Tabla 3 ).

\section{Cobre en la biomasa aérea de las especies prospectadas}

Todas las especies muestreadas presentaron acumulación de $\mathrm{Cu}$ por sobre lo normal (mayor a $20 \mathrm{mg} \mathrm{kg}^{-1}$, Adriano 1986). La mayor concentración de $\mathrm{Cu}$ se encontró en Oenothera affinis con $614 \mathrm{mg} \mathrm{kg}^{-1}$. El resto de las especies presentaron baja o media acumulación de $\mathrm{Cu}$ (Tabla 4).

TABLA 2

Características químicas de los suelos estudiados. Se presentan el rango de valores y las medianas de los parámetros analizados. Concentraciones están expresadas al peso seco

Chemical characteristics of the studied soils. Range of values and medians of analyzed parameters are given. Concentrations are expressed on a dry basis

\begin{tabular}{|c|c|c|c|c|}
\hline Sitio & & $\mathrm{Cu}$ total $\left(\mathrm{mg} \mathrm{kg}^{-1}\right)$ & $\mathrm{Cu}$ soluble $\left(\mathrm{mg} \mathrm{kg}^{-1}\right)$ & $\mathrm{pH}$ \\
\hline \multirow[t]{2}{*}{ Matorral $(n=8)$} & Rango & $92-872$ & $1,1-30$ & $3,9-5,9$ \\
\hline & Mediana & 550 & 23 & 4,5 \\
\hline \multirow[t]{2}{*}{ Escorias $(n=6)$} & Rango & $533-2.790$ & $1,6-44$ & $4,1-5,1$ \\
\hline & Mediana & 1.289 & 26 & 4,8 \\
\hline
\end{tabular}


TABLA 3

Especies identificadas en las áreas de estudio (muestreo de agosto). Se presenta el ciclo de vida $(\mathrm{CV})$, forma de vida (FV), estado fenológico $(\mathrm{EF})$ y origen $(\mathrm{OR}) ; \mathrm{h}=$ hojas, $\mathrm{fl}=$ flores, $\mathrm{fr}=$ frutos, $\mathrm{b}=$ bulbo; $\mathrm{HA}=$ hierba anual, $\mathrm{HP}=$ hierba perenne, $\mathrm{A}=$ arbusto, $\mathrm{HB}=$ hierba bianual; $\mathrm{HC}=$ hemicriptófita, TR = terófita, $\mathrm{NF}=$ nanofanerófita; $\mathrm{I}=$ introducida; $\mathrm{N}=$ nativa; $\mathrm{E}=$ endémica

Species identified in the study areas (August sampling). Life cycle (CV), life form (FV), and phenological stage (EF) are given; $\mathrm{h}=$ leaves, $\mathrm{fl}=$ flowers, $\mathrm{fr}=$ fruits, $\mathrm{b}=$ bulb; $\mathrm{HA}=$ annual herb, $\mathrm{HP}=$ perennial herb $, \mathrm{A}=\mathrm{shrub}, \mathrm{HB}=\mathrm{biannual}$ herb; $\mathrm{HC}=$ hemicryptophyte; $\mathrm{TR}=$ therophyte; $\mathrm{NF}=$ nanophanerophyte; $\mathrm{I}=$ exotic, $\mathrm{N}=$ native; $\mathrm{E}=$ endemic

\begin{tabular}{|c|c|c|c|c|c|}
\hline Familia & Especie & $\mathrm{CV}$ & FV & $\mathrm{EF}$ & $\mathrm{OH}$ \\
\hline \multicolumn{6}{|c|}{ Área de escorias } \\
\hline Aizoaceae & Galenia pubescens Druce & $\mathrm{HP}$ & $\mathrm{HC}$ & $\mathrm{h} / \mathrm{fl}$ & I \\
\hline Asteraceae & Anthemis cotula $\mathrm{L}$. & HA & TR & $\mathrm{h}$ & I \\
\hline Asteraceae & Baccharis salicifolia (R. et P.) Pers. & A & $\mathrm{NF}$ & $\mathrm{h}$ & I \\
\hline Asteraceae & Conyza sp. & HA & TR & $\mathrm{h}$ & - \\
\hline Asteraceae & Pseudognaphalium luteoalbum (L.) Hilliard et B.L. Burtt & HA & TR & $\mathrm{h}$ & I \\
\hline Brassicaceae & Hirschfeldia incana (L.) Lagr.-Fossat & HA & TR & $\mathrm{h}$ & I \\
\hline Chenopodiaceae & Atriplex repanda $\mathrm{L}$. & A & NF & $\mathrm{h}$ & $\mathrm{E}$ \\
\hline Chenopodiaceae & Chenopodium ambrosioides $\mathrm{L}$. & $\mathrm{HP}$ & $\mathrm{HC}$ & $\mathrm{h}$ & $\mathrm{N}$ \\
\hline Malvaceae & Sphaeralcea velutina K. Presl. & A & $\mathrm{NF}$ & $\mathrm{h}$ & $\mathrm{E}$ \\
\hline Onagraceae & Oenothera affinis Cambess. & HB & $\mathrm{HC}$ & $\mathrm{h} / \mathrm{fl}$ & $\mathrm{N}$ \\
\hline Oxalidaceae & Oxalis micrantha Bert. ex Colla & HA & TR & $\mathrm{h} / \mathrm{fl} / \mathrm{fr}$ & $\mathrm{N}$ \\
\hline Papaveraceae & Argemone subfusiformis Ownbey & HA & TR & $\mathrm{h}$ & $\mathrm{N}$ \\
\hline Papaveraceae & Eschscholzia californica Cham. & $\mathrm{HP}$ & $\mathrm{HC}$ & $\mathrm{h} / \mathrm{fl}$ & I \\
\hline Papillionaceae & Melilotus indicus (L.) All. & HA & TR & $\mathrm{h} / \mathrm{fl}$ & $\mathrm{N}$ \\
\hline Poaceae & Distichlis spicata (L.) Greene & $\mathrm{HP}$ & CR & $\mathrm{h}$ & $\mathrm{N}$ \\
\hline Scrophulariaceae & Verbascum virgatum Stockes & НВ & TR & $\mathrm{h}$ & I \\
\hline
\end{tabular}

Área de matorral

Amaryllidaceae

Asteraceae

Asteraceae

Asteraceae

Asteraceae

Brassicaceae

Chenopodiaceae

Malvaceae

Onagraceae

Papaveraceae

Papillionaceae

Poaceae

Portulacaceae

Solanaceae

Scrophulariaceae
Rodophiala advena (Ker-Gawl.) Traub

Antemis cotula $\mathrm{L}$.

Baccharis concava (R. Et P.) Pers.

Baccharis linearis (R. et P.) Pers.

Pseudognaphalium luteoalbum (L.) Hilliard et B.L. Burtt

Hirschfeldia incana (L.) Lagr.-Fossat

Chenopodium ambrosioides $\mathrm{L}$.

Sphaeralcea velutina K. Presl.

Oenothera affinis Cambess.

Eschscholzia californica Cham.

Astragalus amatus $\mathrm{Clos}$

Distichlis spicata (L.) Greene

Calandrinia sp.

Solanum alphonsii Dunal

Verbascum virgatum Stockes

$\begin{array}{llll}\text { HP } & \text { CR } & \text { h/b } & \text { I } \\ \text { HA } & \text { TR } & \text { h/fl } & \text { I } \\ \text { A } & \text { NF } & \text { h } & \text { N } \\ \text { A } & \text { NF } & \text { h } & \text { N } \\ \text { HA } & \text { TR } & \text { h } & \text { I } \\ \text { HA } & \text { TR } & \text { h/fl } & \text { I } \\ \text { HP } & \text { HC } & \text { h } & \text { N }\end{array}$

$\mathrm{HP} \mathrm{HC} \mathrm{h}$ N

A NF $h \quad$ E

HB $\mathrm{HC} h \mathrm{~h}$

HP HC h/fl I

HP HC h/fl E

HP CR h

HA TR h/fl

HP NF h/fl - 


\section{TABLA 4}

Especies identificadas en las áreas de estudio, clasificadas según la concentración de Cu en las biomasas aéreas. Se muestran los promedios de la concentración de $\mathrm{Cu}$ con sus respectivas desviaciones estándar en base a tres repeticiones de una muestra compuesta por dos individuos (muestreo de agosto). Concentraciones están expresadas en peso seco

Species identified in the study areas, classified by copper concentration in the shoots. The average of copper concentration and their respective standard deviations of three replicates of a composed by two individuals sample are shown (August sampling). Concentrations are expressed on a dry weight basis

Área y nivel de acumulación

Media acumulación de Cu (200-600 mg kg-1)

Baja acumulación de $\mathrm{Cu}\left(<200 \mathrm{mg} \mathrm{kg}{ }^{-1}\right)$

Alta acumulación de $\mathrm{Cu}\left(601-1.000 \mathrm{mg} \mathrm{kg}^{-1}\right)$

Media acumulación de Cu (200-600 mg kg-1)

Baja acumulación de $\mathrm{Cu}\left(<200 \mathrm{mg} \mathrm{kg}^{-1}\right)$
Especie

$\mathrm{Cu}$ en la biomasa aérea $\left(\mathrm{mg} \mathrm{kg}^{-1}\right)$
Área de escorias

Baccharis salicifolia

Pseudognaphalium luteoalbum

Argemone subfusiformis

Chenopodium ambrosioides

Atriplex repanda

Oxalis micrantha

Sphaeralcea velutina

Anthemis cotula

Verbascum virgatum

Galenia pubescens

Oenothera affinis

Eschscholzia californica

Distichlis spicata

Conyza sp.

Hirschfeldia incana

Melilotus indicus

Área de matorral

Oenothera affinis

Baccharis linearis

Baccharis concava

Sphaeralcea velutina

Pseudognaphalium luteoalbum

Calandrinia sp.

Solanum alphonsii

Astragalus amatus

Anthemis cotula

Rodophiala advena

Verbascum virgatum

Chenopodium ambrosioides

Hirschfeldia incana

Eschscholzia californica

Distichlis spicata
$452 \pm 26$

$418 \pm 22$

$391 \pm 130$

$368 \pm 92$

$248 \pm 21$

$243 \pm$ -

$208 \pm 13$

$203 \pm-$

$185 \pm 2,5$

$169 \pm 27$

$158 \pm 4,9$

$135 \pm 3,2$

$151 \pm 17$

$83 \pm 4,4$

$73 \pm 16$

$68 \pm 14$

$614 \pm 4,6$

$314 \pm 51$

$255 \pm 29$

$177 \pm 17$

$167 \pm 4,1$

$142 \pm 4,3$

$140 \pm 8,4$

$132 \pm 2,9$

$113 \pm 3,4$

$102 \pm 3,3$

$77 \pm 7,0$

$58 \pm 0,7$

$58 \pm 8,3$

$44 \pm 1,1$

$42 \pm$ - 
Ginocchio (1997) determinó la concentración de cobre foliar en Argemone subfusiformis, Eschscholtzia californica y Baccharis linearis en un estudio sobre la acumulación de $\mathrm{Cu}$ en una comunidad vegetal en las cercanías de la Fundición Ventanas, obteniéndose rangos de concentración de 160-780, 45-196 y 275-453 mg $\mathrm{kg}^{-1}$, respectivamente. Estos rangos de concentración son similares a los encontrados en esta investigación.

Por otra parte, existen antecedentes sobre Hirschfeldia incana, para la cual se reportó una concentración máxima de $355 \mathrm{mg} \mathrm{kg}^{-1}$ en suelos cercanos a una mina en Cataluña, España (suelos con aproximadamente $5.000 \mathrm{mg}$ $\mathrm{kg}^{-1}$ de $\mathrm{Cu}$ total) (Poschenrieder et al. 2001), en contraste con la acumulación de $73 \mathrm{mg} \mathrm{kg}^{-1}$ encontrada en este estudio. Además, Eschscholtzia californica ha sido reportada previamente como especie tolerante a altas concentraciones de $\mathrm{Cu}$ (Vara \& Oliveira 2003), aunque los autores no especificaron las concentraciones de $\mathrm{Cu}$ en el suelo ni en los tejidos. También Distichlis spicata ha sido encontrada en sitios levemente contaminados por cobre en Montana (Estados Unidos de América), en las cercanías de una fundición (suelos con concentraciones de $\mathrm{Cu}$ total de aproximadamente $100 \mathrm{mg} \mathrm{kg}^{-1}$ ) presentando concentraciones de $\mathrm{Cu}$ en la biomasa aérea por sobre $20 \mathrm{mg} \mathrm{kg}^{-1}$ (Prodgers \& Inskeep 1991).

Relación entre las concentraciones de $\mathrm{Cu}$ en biomasa aérea y en el suelo

A pesar que el rango de concentración de $\mathrm{Cu}$ en las plantas fue amplio en cada área de muestreo, las plantas en el área de escorias de fundición presentaron mayores concentraciones de $\mathrm{Cu}$ que las mismas especies muestreadas en el área de matorral (Tabla 4).

El hecho anterior supone una relación entre la absorción de $\mathrm{Cu}$ por las plantas y las fuentes de $\mathrm{Cu}$ en el suelo. La excepción a lo anterior fue $O$. affinis, ya que presentó una mayor concentración de $\mathrm{Cu}$ en el área de matorral que en el área de escorias. Sin embargo, los individuos muestreados se encontraban en distinto estados fenológicos. Específicamente, las muestras de $O$. affinis en el área de matorral se encontraban en estado vegetativo, mientras que las muestras del área de escorias se encontraban en estado reproductivo. El efecto del estado fenológico sobre la concentración de $\mathrm{Cu}$ en las plantas se discute a continuación.

Variación temporal en la concentración de $\mathrm{Cu}$ en la biomasa aérea de algunas especies identificadas

El muestreo realizado en diciembre permitió comparar las concentraciones de cobre en las biomasas aéreas de Chenopodium ambrosioides, Sphaeralcea velutina, Verbascum virgatum, A. subfusiformis y $O$. affinis en diferentes estados de desarrollo: vegetativo (agosto) versus reproductivo (diciembre) (Fig. 2). Para una misma especie, los individuos muestreados en estado de desarrollo vegetativo presentaron concentraciones de cobre mayores que los individuos muestreados en desarrollo de estructuras reproductivas (flores y/o frutos). Específicamente para $A$. subfusiformis y $O$. affinis (especies de hábito bianual o subperenne) se comprobó que la disminución de las concentraciones de $\mathrm{Cu}$ en toda la biomasa aérea, al cambiar el estado fenológico en la planta desde el estado vegetativo al reproductivo, se debió a que las nuevas estructuras desarrolladas presentaron una baja acumulación de $\mathrm{Cu}$ (flores y frutos), a excepción de las flores de $O$. affinis (Tabla 5). Además, las hojas de verano por su menor tiempo de desarrollo presentaron una menor concentración de $\mathrm{Cu}$ que las hojas de otoño, tal como lo demostraron anteriormente Weis et al. (2003). También Navarro \& Navarro (2003) señalan que la absorción de elementos es más rápida e intensa en el período vegetativo, mientras que, en estados posteriores de desarrollo, la absorción tiene menor intensidad, debido al predominio de actividades de síntesis de glúcidos. En definitiva, las nuevas estructuras desarrolladas, de baja acumulación de $\mathrm{Cu}$, causaron que, al considerar toda la biomasa aérea, el promedio de la concentración de $\mathrm{Cu}$ disminuyera.

En estado de senescencia, la concentración de $\mathrm{Cu}$ en la biomasa aérea de $O$. affinis es aún menor que en estado reproductivo. Esto se debe a que la planta pierde la mayor parte de la biomasa de alta concentración de $\mathrm{Cu}$ (hojas de otoño), manteniendo solo las hojas de verano, frutos secos, semillas y el tallo, estructuras que presentan baja concentración de $\mathrm{Cu}$. Además, las hojas de otoño que no han caído presentan también menor concentración de $\mathrm{Cu}$ (Tabla 5), 
llegando solo a un $35 \%$ del valor que tenían en estado vegetativo. Este hecho puede ser producto de una retranslocación parcial de $\mathrm{Cu}$ desde la zona aérea hacia las raíces, al llegar la senescencia, lo que constituye un proceso común de economía de nutrientes en especies perennes y bianuales (Mortvedt et al. 1991), como es el caso de $O$. affinis. Sin embargo, Weis et al. (2003) demostraron, para dos especies palustres: Spartina alterniflora y Phragmithes australis (ambas hierbas perennes rizomatosas) que, en estado de senescencia, $P$. australis retransloca parte del cobre acumulado en las hojas de otoño hacia raíces y rizomas, tal como sucede con $O$. affinis, mientras que $S$. alterniflora lo mantiene en su biomasa aérea (Tabla 5). Este hecho indica que el proceso de retranslocación no afecta a todas las plantas de régimen perenne y debe ser estudiado para cada caso en particular. Marschner (1986) especificó que el proceso de retranslocación se desarrolla solamente en las hojas que emergieron primero en la temporada (en este caso las hojas de otoño), no así en hojas nuevas (en este caso las hojas de verano), tal como sucede en la presente investigación. Otros autores postulan que, en el caso específico del cobre, este tiende a desplazarse desde las hojas viejas a las nuevas y, en algunas especies, también ocurre a nivel de los frutos (Hocking \& Pate 1978, Loué 1988). Sin embargo, esta hipótesis no puede ser confirmada, debido a que en este estudio no se evaluó la movilidad del $\mathrm{Cu}$ en condiciones controladas.

En conclusión, la disminución de las concentraciones de cobre en la biomasa aérea de ambas especies está ligada al cambio de estado fenológico (de vegetativo a reproductivo y a senescencia). La disminución en las concentraciones puede ser explicada por el desarrollo de nuevos órganos no acumuladores, pérdida de biomasa acumuladora y/o retranslocación de cobre a estructuras subterráneas.

Por otra parte, a pesar que los tejidos fueron lavados cuidadosamente, las concentraciones de $\mathrm{Cu}$ podrían estar sobreestimadas, ya que las partículas de suelo pueden permanecer adheridas a la superficie de las hojas y tallo, sobre todo en plantas con gran número de glándulas resiníferas y tricomas, como Baccharis linearis (Ginocchio et al. 2004) y $O$. affinis, respectivamente. Este hecho podría influir tanto en las concentraciones máximas encontradas para estas especies como en las diferencias en estado fenológico (ya que las hojas de otoño han acumulado por más tiempo la contaminación externa).

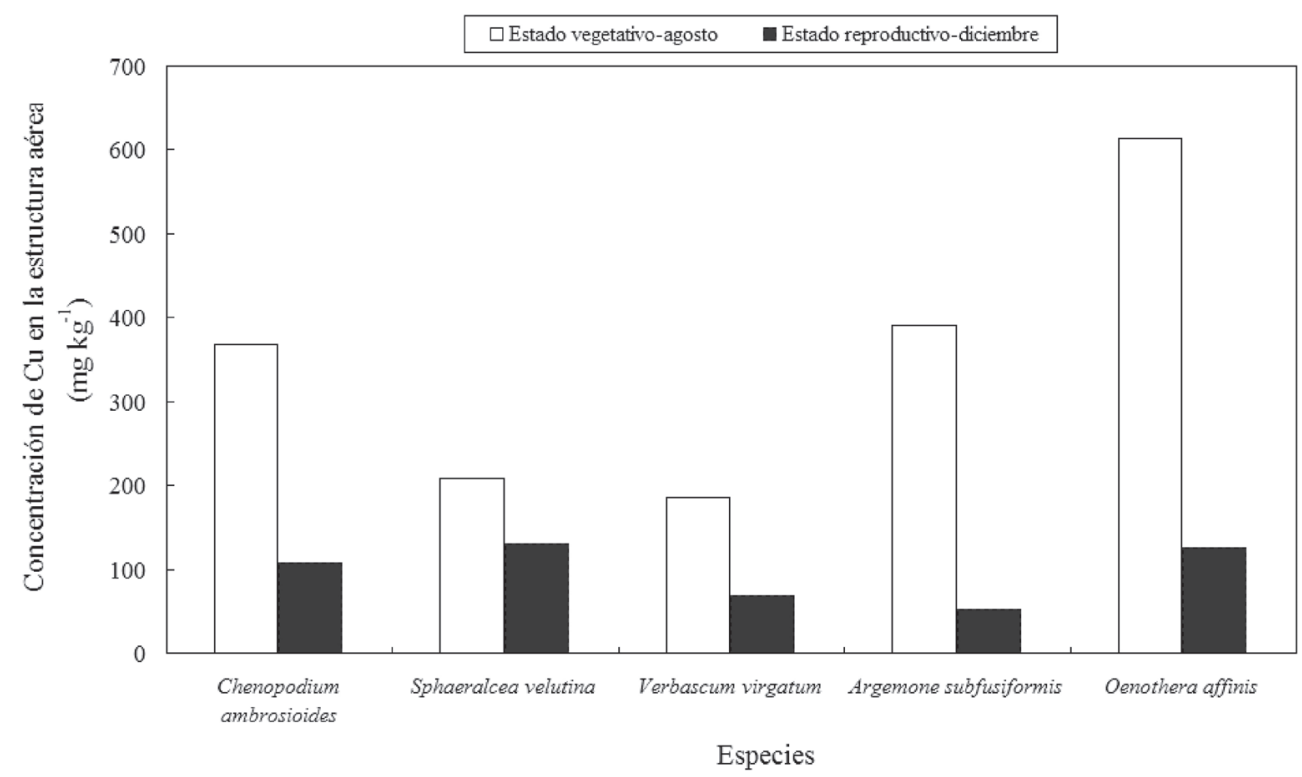

Fig. 2: Variación de la concentración de $\mathrm{Cu}$ en la biomasa aérea de las diferentes especies, en función del estado fenológico de la planta.

Variation in $\mathrm{Cu}$ concentrations in shoots as a function of the plant phenological stage, for different species. 


\section{TABLA 5}

Concentraciones de cobre (expresadas al peso seco) en los diferentes tejidos de la biomasa aérea de A. subfusiformis y $O$. affinis muestreadas en estado vegetativo y reproductivo. Las hojas de otoño corresponden a las primeras que emergieron, las hojas de verano corresponden a las últimas hojas que emergieron. Adicionalmente se entregan datos obtenidos por otros autores. $\mathrm{N} / \mathrm{m}=$ no muestreados, $\mathrm{N} / \mathrm{c}=$ no corresponde al estado fenológico

Copper concentrations (expressed on a dry basis) in different tissues of the above-ground structures of A. subfusiformis and $O$. affinis sampled in vegetative and reproductive stages. The fall leaves correspond to the earlier emerged ones, the summer leaves correspond to the later emerged ones. Additional information obtained by other authors is given. $\mathrm{N} / \mathrm{m}=\mathrm{not}$ sampled; $\mathrm{N} / \mathrm{c}=$ does not correspond to the phenological stage

\begin{tabular}{|c|c|c|c|c|c|c|c|}
\hline \multirow[t]{2}{*}{ Especie } & \multirow[t]{2}{*}{ Estado fenológico } & \multicolumn{6}{|c|}{ Concentración de cobre $\left(\mathrm{mg} \mathrm{kg}^{-1}\right)$} \\
\hline & & $\begin{array}{c}\text { Hojas de } \\
\text { otoño }\end{array}$ & $\begin{array}{c}\text { Hojas de } \\
\text { verano }\end{array}$ & Tallo & Frutos & Flores & $\begin{array}{c}\text { Biomasa } \\
\text { aérea } \\
\text { completa }\end{array}$ \\
\hline \multirow[t]{3}{*}{ Argemone subfusiformis (área de escorias) } & Vegetativo & 391 & $\mathrm{~N} / \mathrm{c}$ & 43 & $\mathrm{~N} / \mathrm{c}$ & $\mathrm{N} / \mathrm{c}$ & $391 \pm 134$ \\
\hline & Reproductivo & 429 & 54,2 & 43 & 36 & 97 & $52 \pm 1,0$ \\
\hline & Senescencia & $\mathrm{N} / \mathrm{m}$ & $\mathrm{N} / \mathrm{m}$ & $\mathrm{N} / \mathrm{m}$ & $\mathrm{N} / \mathrm{m}$ & $\mathrm{N} / \mathrm{m}$ & - \\
\hline \multirow[t]{3}{*}{ Oenothera affinis (área matorral) } & Vegetativo & 614 & $\mathrm{~N} / \mathrm{c}$ & 50 & $\mathrm{~N} / \mathrm{c}$ & $\mathrm{N} / \mathrm{c}$ & $614 \pm 25$ \\
\hline & Reproductivo & 524 & 318 & 50 & 78 & 493 & $153 \pm 13$ \\
\hline & Senescencia & 217 & 338 & 50 & 104 & $\mathrm{~N} / \mathrm{c}$ & $101 \pm 19$ \\
\hline Spartina alterniflora (Weis et al. 2003) & Senescencia & 250 & 108 & $\mathrm{~N} / \mathrm{m}$ & $\mathrm{N} / \mathrm{c}$ & $\mathrm{N} / \mathrm{c}$ & $175 \pm 93$ \\
\hline Phragmithes australis (Weis et al. 2003) & Senescencia & 80 & 150 & $\mathrm{~N} / \mathrm{m}$ & $\mathrm{N} / \mathrm{c}$ & $\mathrm{N} / \mathrm{c}$ & $110 \pm 36$ \\
\hline
\end{tabular}

\section{Implicancias para la fitoextracción}

Los resultados de esta prospección indican que en el área de Los Maitenes existen especies capaces de tolerar y acumular distintas concentraciones de $\mathrm{Cu}$. Las 22 especies identificadas en el área se clasifican como "pseudometalofitas" (Poschenrieder et al. 2001), ya que son poblaciones de especies comunes en Chile que crecen con mayor frecuencia en suelos no contaminados (e.g., Marticorena \& Quezada 1985, Matthei 1995).

Para el caso del cobre, aunque no se identificaron hiperacumuladoras, se identificó una población de Oenothera affinis con altas concentraciones de $\mathrm{Cu}$. Esta especie se desarrolla frecuentemente en orillas de caminos como también en praderas degradadas, además, es resistente a la sequía y necesita escasa mantención (Matthei 1995). Estas características la hacen ser una candidata potencial para desarrollar un proceso de fitoextracción a gran escala, donde es preferible utilizar plantas que no requieran manejos agronómicos. Sin embargo, es necesario determinar su producción de biomasa y comprobar su real capacidad de acumulación de $\mathrm{Cu}$, evitando la contaminación externa de los tejidos. Actualmente, se están cultivando individuos de $O$. affinis y $B$. linearis a partir de semillas en un sitio libre de contaminación atmosférica (ex situ), utilizando suelos provenientes de los sectores de muestreo de esta investigación. De esta forma, a futuro se determinará la absorción real de la planta y se estimará el grado de contaminación externa por partículas adheridas a los tricomas, por comparación con los resultados de esta investigación.

Los resultados referentes a la distribución de $\mathrm{Cu}$ a lo largo del desarrollo de la planta evidencian la necesidad de realizar estudios más detallados para entender la dinámica de la absorción, acumulación y retranslocación de $\mathrm{Cu}$ en las distintas estructuras de la planta. El conocimiento de esta dinámica beneficiaría directamente la eficiencia de un proceso de fitoextracción, ya que se podría determinar la etapa exacta del desarrollo en que la planta concentra la mayor cantidad de metal en su biomasa aérea, y por tanto, el momento ideal para su cosecha. 


\section{AGRADECIMIENTOS}

Este estudio fue financiado por el proyecto FONDECYT 1050403. Los autores agradecen los comentarios de Rosanna Ginocchio.

\section{LITERATURA CITADA}

ADRIANO D (1986) Trace elements in terrestrial environments. Springer-Verlag, New York, New York, USA. 533 pp.

BAKER AJM \& RR BROOKS (1989) Terrestrial higher plants which hyperaccumulate metallic elements: A review of their distribution, ecology and phytochemistry. Biorecovery 1: 81-108.

BRUNO C (1992) El viento y el relieve como factores geográficos determinantes en la dispersión de contaminantes derivados de los procesos productivos del cobre (comuna de Puchuncaví, V Región). Tesis de Magíster en Asentamientos Humanos y Medio Ambiente, Pontificia Universidad Católica de Chile, Santiago, Chile. $103 \mathrm{pp}$.

DE GREGORI I, E FUENTES, M ROJAS, H PINOCHET \& M POTIN-GAUTIER (2003) Monitoring of copper, arsenic and antimony levels in agricultural soils and non-impacted mining activities, from three regions in Chile. Journal of Environmental Monitoring 5: 287-295.

GINOCCHIO R (1997) Aplicabilidad de los modelos de distribución espacio-temporales de la vegetación en ecosistemas sujetos a procesos de contaminación. Tesis para optar al grado de Doctor en Ciencias Biológicas, Mención Ecología, Departamento de Ecología, Pontificia Universidad Católica de Chile, Santiago, Chile. 209 pp.

GINOCCHIO R (2000) Effects of a copper smelter on a grassland community in the Puchuncaví Valley, Chile. Chemosphere 41: 15-23.

GINOCCHIO R (2004) Solución vegetal. Induambiente (Chile) 67: 30-32.

GINOCCHIO R \& A BAKER (2004) Metalofitas en América Latina: un recurso biológico y genético único poco conocido y estudiado en la región. Revista Chilena de Historia Natural 77: 185-194.

GINOCCHIO R, G CARVALLO, I TORO, E BUSTAMANTE, Y SILVA \& N SEPÚLVEDA (2004) Micro-spatial variation of soil metal pollution and plant recruitment near a copper smelter in central Chile. Environmental Pollution 127: 343-352.

HOCKING PJ \& JS PATE (1978) Accumulation and distribution of mineral elements on the annual lupins Lupinus albus L. and Lupinus angustifolius L. Australian Journal of Agricultural Research 29: 267-280.

JAFFRÉ T, W KERSTEN, RR BROOKS \& R REEVES (1979) Nickel uptake by Flacourticeae of New Caledonia. Proceedings of the Royal Society of London, Series B, Biological Sciences 205: 385394.

JOHNSTON MN (1947) Astragalus in Argentina, Bolivia and Chile. Journal of the Arnold Arboretum 28: 384-385.
KABATA-PENDIAS A (2004) Soil-plant transfer of trace elements: an environmental issue. Geoderma 122: 143-149.

LEUENBERGER BE \& U EGGLI (2002) Galenia pubescens (Aizoaceae), new to the South American flora. Botanische Jahrbucher Systematik 123: 441445.

LOUÉ A (1988) Los microelementos en la agricultura. Ediciones Mundi-Prensa, Madrid, España. 354 pp.

MALAISSE F, A BAKER \& S RUELLE (1999) Diversity of plant communities and leaf heavy metal content at Luiswishi copper/cobalt mineralization, Upper Katanga, Democratic Republic of Congo. Biotechnology, Agronomy, Society and Environment 3: 104-114.

MARSCHNER H (1986) Mineral nutrition of higher plants. Academic Press, New York, New York, USA. 674pp.

MARTICORENA C \& M QUEZADA (1985) Flora vascular de Chile. Gayana Botánica (Chile) 42: 13 157.

MATTHEI J (1995) Manual de las malezas que crecen en Chile. Alfabeta Impresores, Santiago, Chile. 537 pp.

MAXWELL J (1968) Rock and mineral analysis Interscience Publishers, New York, New York, USA. 584 pp.

MCGRATH SP, CM SIDOLI, AJ BAKER \& RD REEVES (1993) The potential for use of metal-accumulating plants for the in situ decontamination of metalpolluted soils. En: Eijsackers HJ \& T Hamers (eds) Integrated soil and sediment research: a basis for proper protection: 673-677. Kluwer Academic Publishers, Dordrecht, The Netherlands.

MORTVEDT JJ, PM GIORDANO \& WL LINDSAY (1991) Micronutrientes en agricultura. AGT Editores, Ciudad de México, México. 354 pp.

NAVARRO S \& G NAVARRO (2003) Química agrícola: el suelo y los elementos químicos esenciales para la vida vegetal. Ediciones Mundi-Prensa, Madrid, España. $488 \mathrm{pp}$

NAVAS LE (1979) Flora de la cuenca de Santiago de Chile. Ediciones de la Universidad de Chile, Santiago, Chile. 187 pp.

POLLARD JA, KD POWELL, FA HARPER \& JAC SMITH (2002) The genetic basis of metal hyperaccumulation in plants. Critical Reviews in Plant Sciences 21: 539-566.

POSCHENRIEDER C, J BECH, M LLUGANY, A PACE, E FENÉS \& J BARCELÓ (2001) Copper in plant species in a copper gradient in Catalonia (north east Spain) and their potential for phytoremediation. Plant and Soil 230: 247-256.

PRODGERS RA \& WP INSKEEP (1991) Heavy metal tolerance in inland saltgrass (Distichlis spicata). Great Basin Naturalist 51: 271-278.

REEVES R \& N ADIGÜZEL (2004) Rare plants and niquel accumulator from Turkish serpentine soils, with special reference to Centaurea species. Turkish Journal of Botany 28: 147-153.

SOON YK (1998) Determination of arsenic and mercury in plant tissue. En: Kalra YP (ed) Handbook of reference methods for plant analysis: 183-192. CRC Press, Washington, District of Columbia, USA.

TANG S, B-M WILKE \& JW HUANG (1999) The uptake of copper by plants dominantly growing on copper mining spoils along the Yangtze river, the 
People's Republic of China. Plant and Soil 209: 225-232.

VARA MN \& HM OLIVEIRA (2003) Metal hyperaccumulation in plants: biodiversity prospecting for phytoremediation technology Electronic Journal of Biotechnology 6: 285-310.

Editor Asociado: José Miguel Fariña

Recibido el 9 de noviembre de 2006; aceptado el 3 de enero 2008
WEIS J, L WINDHAM \& P WEIS (2003) Patterns of metal accumulation in leaves of the tidal marsh plants Spartina alterniflora Loisel and Phragmites australis (Cav. Trin ex Steud.) over the growing season. Wetlands 23: 459-465. 
